\title{
Efficacy of budesonide/glycopyrronium/
}

Shigeo Muro ${ }^{1 *} \mathbb{D}$, Hisatoshi Sugiura ${ }^{2}$, Patrick Darken ${ }^{3}$ and Paul Dorinsky ${ }^{4}$

\begin{abstract}
Background: In the Phase III KRONOS study, triple therapy with budesonide/glycopyrronium/formoterol fumarate metered dose inhaler (BGF MDI) was shown to reduce exacerbations and improve lung function versus glycopyrronium/formoterol fumarate dihydrate (GFF) MDI in patients with moderate-to-very severe chronic obstructive pulmonary disease (COPD). However, whether the benefits related to the ICS component of BGF are driven by patients with high blood eosinophil counts (EOS) and/or airway reversibility has not been previously studied.

Methods: KRONOS was a Phase III, double-blind, parallel-group, multicenter, randomized, controlled study of patients with moderate-to-very-severe COPD. Patients were randomized 2:2:1:1 to receive BGF 320/14.4/10 $\mu \mathrm{g}$, GFF 14.4/10 $\mu \mathrm{g}$, budesonide/formoterol fumarate dihydrate (BFF) MDI 320/10 $\mu \mathrm{g}$ via a single Aerosphere inhaler, or open-label budesonide/formoterol fumarate dihydrate dry powder inhaler 400/12 $\mu \mathrm{g}$ (BUD/FORM DPI; Symbicort Turbuhaler) twice-daily for 24 weeks. Efficacy outcomes included in this post-hoc analysis were change from baseline in morning pre-dose trough $\mathrm{FEV}_{1}$ over weeks 12-24 and the rate of moderate-to-severe and severe COPD exacerbations. Adverse events in the non-reversible subgroup are also reported.

Results: Of 1896 patients analyzed, 948 (50\%) were non-reversible and had EOS $<300$ cells $/ \mathrm{mm}^{3}$. In this group, BGF significantly improved morning pre-dose trough $\mathrm{FEV}_{1}$ versus BFF and BUD/FORM (least squares mean treatment difference, 95\% confidence interval [CI] $69 \mathrm{~mL}[39,99]$, unadjusted $p<0.0001$ and $51 \mathrm{~mL}[20,81]$, unadjusted $p=0.0011$, respectively) and was comparable to GFF. BGF also significantly reduced annual moderate-to-severe exacerbation rates versus GFF (rate ratio $[95 \% \mathrm{CI}] 0.53[0.37,0.76]$, unadjusted $p=0.0005$ ), with numerical reductions observed versus BFF and BUD/FORM. These results were similar for the overall study population. Safety findings were generally similar between non-reversible patients with EOS $<300$ cells $/ \mathrm{mm}^{3}$ and the overall population.
\end{abstract}

Conclusions: In patients with moderate-to-very-severe COPD without airway reversibility and EOS $<300 \mathrm{cells} / \mathrm{mm}^{3}$, BGF significantly improved morning pre-dose trough FEV ${ }_{1}$ versus BFF and BUD/FORM and significantly reduced the

\footnotetext{
*Correspondence: smuro@naramed-u.ac.jp

${ }^{1}$ Department of Respiratory Medicine, Nara Medical University Graduate

School of Medicine, 840 Shijo-cho, Kashihara-shi, Nara 634-8521, Japan

Full list of author information is available at the end of the article
} permitted by statutory regulation or exceeds the permitted use, you will need to obtain permission directly from the copyright holder. To view a copy of this licence, visit http://creativecommons.org/licenses/by/4.0/. The Creative Commons Public Domain Dedication waiver (http://creativecommons.org/publicdomain/zero/1.0/) applies to the data made available in this article, unless otherwise stated in a credit line to the data. 
rate of moderate-to-severe exacerbations versus GFF. These findings demonstrate that BGF can provide benefits for a broad range of patients with COPD, and that the overall findings of the KRONOS primary analysis were not driven by patients with reversible airflow obstruction or high eosinophil counts.

Trial registration ClinicalTrials.gov, NCT02497001. Registered 14 July 2015, https://clinicaltrials.gov/ct2/show/NCT02 497001

Keywords: COPD, Asthma-like features, Triple therapy, Budesonide, Glycopyrrolate, Formoterol fumarate, KRONOS, Pulmonary function, Exacerbation

\section{Background}

The Global Initiative for Chronic Obstructive Lung Disease (GOLD) recommends treatment with a triple combination of an inhaled corticosteroid (ICS), a long-acting muscarinic antagonist (LAMA), and a long-acting $\beta_{2^{-}}$ agonist (LABA) for patients with COPD who experience further exacerbations despite LAMA/LABA or ICS/ LABA therapy, or persistent breathlessness on ICS/LABA therapy [1]. However, the Japanese Respiratory Society (JRS) only recommends ICS-containing treatments for the management of patients with COPD with additional features associated with asthma (also known as asthmaCOPD overlap or ACO), which include airway reversibility (post-bronchodilator response in forced expiratory volume in $1 \mathrm{~s}\left[\mathrm{FEV}_{1}\right] \geq 200 \mathrm{~mL}$ and $\geq 12 \%$ from baseline values) and a blood eosinophil count (EOS) $\geq 300$ cells/ $\mathrm{mm}^{3}[2,3]$. GOLD acknowledges that asthma and COPD can share common clinical features, such as some degree of airway reversibility and high EOS, and recommends that pharmacotherapy should primarily follow asthma guidelines upon a concurrent diagnosis of asthma and COPD; however, pharmacological and non-pharmacological treatment of COPD may also be needed [1].

The KRONOS study (NCT02497001) was a 24-week, Phase III, randomized, double-blind, parallel-group trial, investigating the efficacy and safety of the triple fixeddose combination budesonide/glycopyrronium/formoterol fumarate dihydrate metered dose inhaler (BGF $\mathrm{MDI}$ ) versus the corresponding dual therapies glycopyrronium/formoterol fumarate dihydrate (GFF) MDI and, budesonide/formoterol fumarate dihydrate (BFF) MDI, and open-label budesonide/formoterol fumarate dihydrate dry powder inhaler (BUD/FORM DPI) in patients from Canada, China, Japan, and the US [4]. Treatment with BGF was shown to provide benefits on lung function, symptoms, and exacerbations versus dual therapies, and was well tolerated in patients with moderate-to-very severe COPD.

The efficacy of triple therapy has previously been reported in COPD populations that included patients with some features of asthma [5, 6]. Improvements in exacerbation rates following ICS therapy in COPD have been observed to occur over a broad range of blood
EOS levels but with the magnitude of effect increasing as blood EOS increases [7]. However, lung function responses to ICS are driven by EOS in COPD [8] and benefits of ICS on lung function are greater in patients with asthma than COPD [9]. In this regard, it has also been suggested that the efficacy of ICS-containing treatments in COPD trials may be driven by patients with a history of asthma [5]. While KRONOS did not enroll patients with a current diagnosis of asthma, those with a previous history of asthma were not excluded, and some patients had certain disease characteristics which, while present in many patients with COPD, can also be clinical features of asthma (airway reversibility and/or elevated EOS) [1]. Therefore, this post-hoc analysis of the KRONOS study aimed to evaluate lung function and exacerbations in patients with moderate-to-very severe COPD who did not have airway reversibility and who had EOS $<300$ cells $/ \mathrm{mm}^{3}$, to assess whether the benefits of BGF were driven by patients with some clinical features that overlap with asthma.

\section{Methods}

\section{Study design}

Details of the KRONOS study design have been previously reported [4]. Patients were randomized 2:2:1:1 to receive BGF $320 / 14.4 / 10 \mu \mathrm{g}$, GFF $14.4 / 10 \mu \mathrm{g}$, or BFF $320 / 10 \mu \mathrm{g}$ via a single Aerosphere inhaler, or open-label BUD/FORM DPI 400/12 $\mu \mathrm{g}$ (Symbicort Turbuhaler) for 24 weeks of twice-daily treatment. As BFF MDI is not an approved therapy for COPD, BUD/FORM DPI was included in the study as an approved active comparator for BGF MDI. As the administration instructions for DPIs and MDIs are markedly different, BUD/FORM DPI was administered in an open-label fashion to avoid the need for a double-dummy design that may have impacted proper device use. Of note, in BGF, GFF, and BFF the doses of glycopyrronium and formoterol fumarate dihydrate are equivalent to glycopyrrolate $18 \mu \mathrm{g}$ and formoterol fumarate $9.6 \mu \mathrm{g}$, respectively.

The study was done in accordance with Good Clinical Practice, including the Declaration of Helsinki. The protocol and informed consent form were approved by appropriate institutional review boards or independent 
ethics committees. All patients provided written informed consent before screening.

\section{Study population}

Inclusion criteria have been previously reported [4]. In brief, eligible patients were $40-80$ years of age, were current/former smokers (smoking history of $\geq 10$ packyears), had moderate-to-very severe airflow limitation (post-bronchodilator $\mathrm{FEV}_{1} \geq 25 \%$ and $<80 \%$ of predicted normal values using appropriate reference norms [e.g. for Japanese patients, JRS reference equations were used] $[10,11])$, and a COPD Assessment Test (CAT) score $\geq 10$, despite receiving $\geq 2$ inhaled maintenance therapies for $\geq 6$ weeks before screening. In addition, there was no requirement for a history of COPD exacerbations in the year before study entry.

Exclusion criteria included a current diagnosis of asthma, any clinically significant respiratory disease other than COPD, or any other clinically significant uncontrolled non-respiratory disease that could influence study results.

\section{Efficacy and safety outcomes}

The primary and secondary endpoints have been reported [4]. The results presented here focus on the effects of the ICS component (budesonide) of BGF on the primary endpoint of trough $\mathrm{FEV}_{1}$ over weeks $12-24$, and rate of moderate-to-severe and severe COPD exacerbations. Adverse events in the non-reversible subgroup are also reported.

\section{Statistical analyses}

Efficacy data were analyzed in the modified intentionto-treat (mITT) population, which included data from all patients obtained prior to discontinuation from treatment. For this post-hoc analysis, outcomes were analyzed in patients without airway reversibility (change in $\mathrm{FEV}_{1}<12 \%$ or $<200 \mathrm{~mL}$ after administration of albuterol) and with EOS $<300$ cells $/ \mathrm{mm}^{3}$. Results for the efficacy endpoints in all enrolled patients, i.e. with any level of airway reversibility and with no restriction on eosinophil count are also presented for reference.

Change from baseline in morning pre-dose trough $\mathrm{FEV}_{1}$ over weeks $12-24$ was analyzed using a linear repeated measures model including treatment, visit, treatment by visit interaction, and ICS use at screening as categorical variables, and baseline $\mathrm{FEV}_{1}$, baseline eosinophil count, and percent reversibility to albuterol as continuous covariates.

The rate of moderate and/or severe exacerbations was analyzed using negative binomial regression, with adjustment for baseline post-bronchodilator percent-predicted $\mathrm{FEV}_{1}$ and baseline eosinophil count as continuous covariates and baseline COPD exacerbation history $(0,1, \geq 2)$, country, and ICS use at screening as categorical covariates. Time at risk of experiencing an exacerbation was used as an offset variable in the model. As this was a post-hoc analysis, no adjustment was made for multiplicity for the subgroup analyses.

Safety variables were summarized descriptively in the safety population, which included all randomized patients who received at least one dose of treatment.

\section{Results}

Half $(948 / 1896)$ of the overall mITT population did not show reversibility to albuterol and had EOS $<300$ cells/ $\mathrm{mm}^{3}$. A total of $1071 / 1896$ (56.5\%) were non-reversible to albuterol and $822 / 1896$ (43.4\%) were reversible to albuterol; 112/1896 (5.9\%) were reversible to albuterol and had $E O S \geq 300$ cells $/ \mathrm{mm}^{3}$. Of note, $1361 / 1896$ $(71.8 \%)$ patients were receiving ICS treatment at screening.

For patients who were non-reversible with EOS $<300$ cells $/ \mathrm{mm}^{3}$, demographic and disease characteristics were generally similar across treatment groups, and similar to that of the overall mITT population. Exceptions were median EOS and mean airway reversibility, which, as expected based on subgroup definition criteria, were greater in the overall population (Table 1). Patients who were non-reversible and had EOS $<300$ cells $/ \mathrm{mm}^{3}$ had a mean age of 65.7 years, $67.0 \%$ were male, $73.2 \%$ reported having no exacerbations in the previous 12 months, and $70.7 \%$ were using ICS at screening. In addition, the mean (standard deviation) post-bronchodilator $\mathrm{FEV}_{1} /$ forced vital capacity ratio (FVC) was $0.48(0.11)$ in non-reversible patients with EOS $<300$ cells $/ \mathrm{mm}^{3}$ and $0.49(0.11)$ in the patients who were excluded from this analysis (due to reversibility and/or EOS $\geq 300$ cells $/ \mathrm{mm}^{3}$ ); $\mathrm{FEV}_{1} / \mathrm{FVC}$ ratio was $0.48(0.11)$ for the overall population.

\section{Efficacy Lung function}

In the overall mITT population, there were significant improvements in morning pre-dose trough $\mathrm{FEV}_{1}$ over weeks 12-24 for BGF versus GFF, BFF, and BUD/FORM (Table 2).

In non-reversible patients with EOS $<300$ cells $/ \mathrm{mm}^{3}$, similar changes from baseline were observed in morning pre-dose trough $\mathrm{FEV}_{1}$ over weeks $12-24$ with BGF and GFF (least squares mean [LSM], 95\% confidence intervals [95\% CI] $97 \mathrm{~mL}[80,115]$ and $102 \mathrm{~mL}$ [84, 120], respectively; Fig. 1, Table 2). However, BGF significantly improved morning pre-dose trough $\mathrm{FEV}_{1}$ over weeks 12-24 compared with BFF and BUD/FORM (LSM 
Table 1 Demographic and baseline disease characteristics (mITT population)

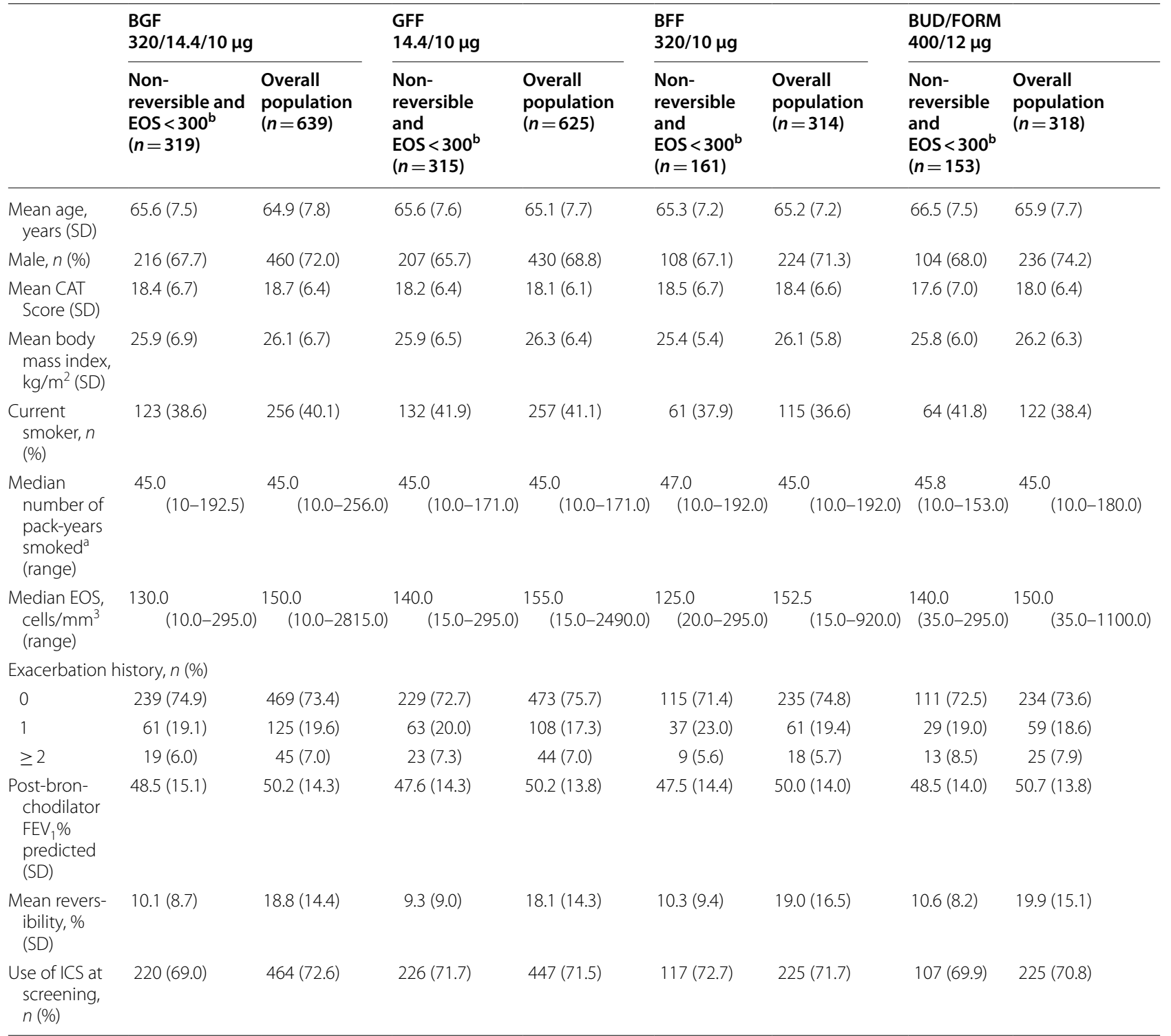

Overall population data from [4]

BFF, budesonide/formoterol fumarate dihydrate; BGF, budesonide/glycopyrronium/formoterol fumarate dihydrate; BUD/FORM DPI, budesonide/formoterol fumarate dihydrate dry powder inhaler; CAT, COPD Assessment Test; COPD, chronic obstructive pulmonary disease; EOS, blood eosinophil count; FEV ${ }_{1}$, forced expiratory volume in $1 \mathrm{~s}$; GFF, glycopyrronium/formoterol fumarate dihydrate; ICS, inhaled corticosteroid; MDI, metered dose inhaler; mITT, modified intent-to-treat; SD, standard deviation

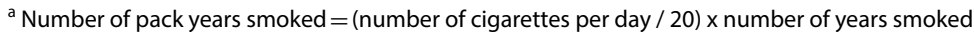

${ }^{\mathrm{b}}$ Patients with airways not reversible to albuterol and $\mathrm{EOS}<300$ cells $/ \mathrm{mm}^{3}$

treatment difference [95\% CI] $69 \mathrm{~mL}[39,99]$, unadjusted $p<0.0001$ and $51 \mathrm{~mL}[20,81]$, unadjusted $p=0.0011$, respectively; Fig. 1, Table 2).

Data for the overall non-reversible population (all patients without reversibility to albuterol, regardless of eosinophil count) and the whole reversible population are presented in Additional file 1: Table S1. Across all treatments, greater improvements in lung function were observed in patients with reversibility compared to those without.

\section{Moderate-to-severe exacerbations}

In the overall mITT population, BGF resulted in a significant reduction in annualized moderate-tosevere exacerbations versus GFF, and numerical reductions versus BFF and BUD/FORM (Table 2). In 
Table 2 Efficacy endpoints (mITT population; efficacy estimand)

\begin{tabular}{|c|c|c|c|c|}
\hline & $\begin{array}{l}\text { BGF } \\
320 / 14.4 / 10 \mu \mathrm{g}\end{array}$ & $\begin{array}{l}\text { GFF } \\
14.4 / 10 \mu \mathrm{g}\end{array}$ & $\begin{array}{l}\mathrm{BFF} \\
320 / 10 \mu \mathrm{g}\end{array}$ & $\begin{array}{l}\text { BUD/FORM } \\
400 / 12 \mu g\end{array}$ \\
\hline \multicolumn{5}{|c|}{ Change from baseline in morning pre-dose trough $\mathrm{FEV}_{1}(\mathrm{~mL})$ over weeks 12-24 } \\
\hline \multicolumn{5}{|c|}{ Overall population } \\
\hline n & 592 & 559 & 278 & 288 \\
\hline LSM (SE) & $138(7.0)$ & $118(7.1)$ & $61(9.9)$ & $76(9.8)$ \\
\hline \multicolumn{5}{|l|}{ BGF versus comparators } \\
\hline LSM difference $(95 \% \mathrm{Cl})$ & - & $20(1,39)$ & $77(53,100)$ & $62(38,85)$ \\
\hline$p$-value & - & 0.0424 & $<0.0001$ & $<0.0001$ \\
\hline \multicolumn{5}{|c|}{ Patients not reversible to albuterol, EOS $<300$ cells $/ \mathrm{mm}^{3}$} \\
\hline$n$ & 285 & 277 & 138 & 140 \\
\hline LSM (SE) & $97(9.0)$ & $102(9.2)$ & $28(12.8)$ & $46(12.8)$ \\
\hline \multicolumn{5}{|l|}{ BGF versus comparators } \\
\hline LSM difference (95\% Cl) & - & $-5(-29,20)$ & $69(39,99)$ & $51(20,81)$ \\
\hline$p$-value & - & 0.7041 & $<0.0001$ & 0.0011 \\
\hline \multicolumn{5}{|l|}{ Rate of moderate-to-severe exacerbations } \\
\hline \multicolumn{5}{|l|}{ Overall population } \\
\hline n & 639 & 625 & 314 & 318 \\
\hline Patients with exacerbations, $n(\%)$ & $108(16.9)$ & $157(25.1)$ & $65(20.7)$ & $61(19.2)$ \\
\hline Adjusted rate per year & 0.46 & 0.95 & 0.56 & 0.55 \\
\hline \multicolumn{5}{|l|}{ BGF versus comparators } \\
\hline Rate ratio $(95 \% \mathrm{Cl})$ & - & $0.48(0.37,0.64)$ & $0.82(0.58,1.17)$ & $0.83(0.59,1.18)$ \\
\hline$p$-value & - & $<0.0001$ & 0.2792 & 0.3120 \\
\hline \multicolumn{5}{|c|}{ Patients not reversible to albuterol, EOS $<300$ cells $/ \mathrm{mm}^{3}$} \\
\hline$n$ & 319 & 315 & 161 & 153 \\
\hline Patients with exacerbations, $n(\%)$ & $56(17.6)$ & $80(25.4)$ & $37(23.0)$ & $39(25.5)$ \\
\hline Adjusted rate per year & 0.46 & 0.87 & 0.56 & 0.68 \\
\hline \multicolumn{5}{|l|}{ BGF versus comparators } \\
\hline Rate ratio $(95 \% \mathrm{Cl})$ & - & $0.53(0.37,0.76)$ & $0.81(0.51,1.29)$ & $0.67(0.43,1.04)$ \\
\hline$p$-value & - & 0.0005 & 0.3770 & 0.0756 \\
\hline \multicolumn{5}{|l|}{ Rate of severe exacerbations } \\
\hline \multicolumn{5}{|l|}{ Overall population } \\
\hline n & 639 & 625 & 314 & 318 \\
\hline Patients with exacerbations, $n(\%)$ & $17(2.7)$ & $33(5.3)$ & $9(2.9)$ & $11(3.5)$ \\
\hline Adjusted rate per year & 0.05 & 0.13 & 0.05 & 0.07 \\
\hline \multicolumn{5}{|l|}{ BGF versus comparators } \\
\hline Rate ratio $(95 \% \mathrm{Cl})$ & - & $0.36(0.18,0.70)$ & $0.85(0.34,2.13)$ & $0.69(0.29,1.61)$ \\
\hline$p$-value & - & 0.0026 & 0.7363 & 0.3861 \\
\hline \multicolumn{5}{|c|}{ Patients not reversible to albuterol, EOS $<300$ cells $/ \mathrm{mm}^{3}$} \\
\hline$n$ & 319 & 315 & 161 & 153 \\
\hline Patients with exacerbations, $n$ (\%) & $11(3.4)$ & $20(6.3)$ & $7(4.3)$ & $3(2.0)$ \\
\hline Adjusted rate per year & 0.07 & 0.18 & 0.10 & 0.03 \\
\hline \multicolumn{5}{|l|}{ BGF versus comparators } \\
\hline Rate ratio $(95 \% \mathrm{Cl})$ & - & $0.40(0.17,0.94)$ & $0.74(0.24,2.30)$ & $2.10(0.50,8.81)$ \\
\hline$p$-value & - & 0.0365 & 0.6057 & 0.3096 \\
\hline
\end{tabular}

Overall population moderate/severe data from [4]

Treatments were compared adjusting for baseline post-bronchodilator percent predicted $\mathrm{FEV}_{1}$ and baseline eosinophil count as continuous covariates and baseline COPD exacerbations history $(0,1, \geq 2)$, country, and ICS use at screening as categorical covariates using negative binomial regression. Time at risk of experiencing an exacerbation was used as an offset variable in the model

BFF, budesonide/formoterol fumarate dihydrate; BGF, budesonide/glycopyrronium/formoterol fumarate dihydrate; BUD/FORM DPI, budesonide/formoterol fumarate dihydrate dry powder inhaler; $\mathrm{Cl}$, confidence interval; EOS, blood eosinophil count; FEV , forced expiratory volume in $1 \mathrm{~s}$; GFF, glycopyrronium/formoterol fumarate dihydrate; LSM, least squares mean; MDI, metered dose inhaler; mITT, modified intent-to-treat; SE, standard error 


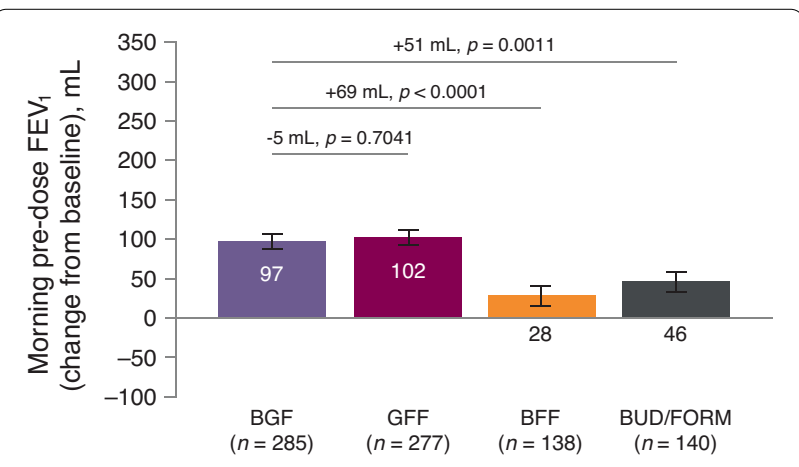

Fig. 1 Change in morning pre-dose trough $\mathrm{FEV}_{1}$ over 12-24 weeks (patients with non-reversible airways and EOS $<300$ cells $/ \mathrm{mm}^{3}$ ). Bars are LSM (95\% SE). Data analyzed in the mITT population using an efficacy estimand. Treatment comparisons are RR (95\% Cl). BFF, budesonide/formoterol fumarate dihydrate; BGF, budesonide/ glycopyrronium/formoterol fumarate dihydrate; BUD/FORM, budesonide/formoterol fumarate dihydrate; FEV , forced expiratory volume in $1 \mathrm{~s}$; GFF, glycopyrronium/formoterol fumarate dihydrate; LSM, least squares mean; mITT, modified intent-to-treat; SE, standard error

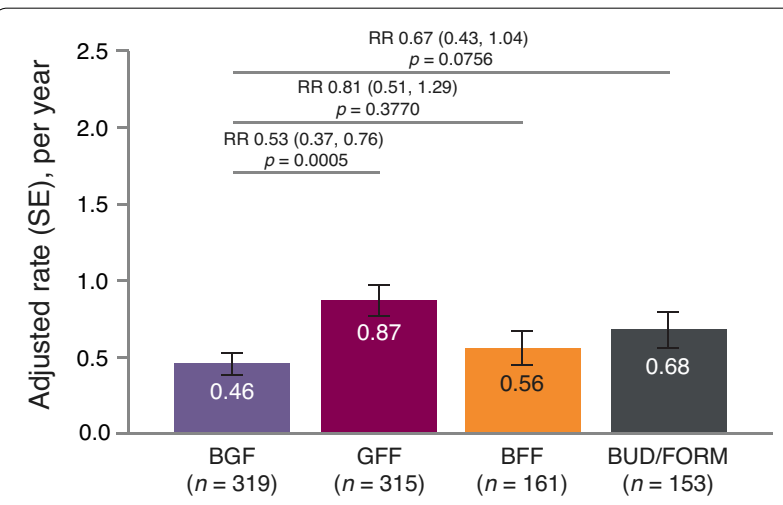

Fig. 2 Adjusted rate of moderate or severe COPD exacerbations (patients with non-reversible airways and $\mathrm{EOS}<300$ cells $/ \mathrm{mm}^{3}$ ). Bars are adjusted rate $( \pm \mathrm{SE})$. Treatment comparisons are RR $(95 \% \mathrm{Cl})$. Data analyzed in the MITT population using an efficacy estimand. BFF, budesonide/formoterol fumarate dihydrate; BGF, budesonide/ glycopyrronium/formoterol fumarate dihydrate; BUD/FORM, budesonide/formoterol fumarate dihydrate; $\mathrm{Cl}$, confidence interval; COPD, chronic obstructive pulmonary disease; EOS, blood eosinophil count; GFF, glycopyrronium/formoterol fumarate dihydrate; mITT, modified intent-to-treat; RR, rate ratio; SE, standard error

non-reversible patients with EOS $<300$ cells $/ \mathrm{mm}^{3}$, BGF significantly reduced annualized moderate-to-severe exacerbation rates versus GFF (adjusted rate ratio [RR], [95\% CI] $0.53[0.37,0.76] p=0.0005$; Fig. 2; Table 2) and there were numerical reductions in annual moderate-to-severe exacerbation rates versus BFF and BUD/
FORM (adjusted RR [95\% CI] $0.81[0.51,1.29]$ and 0.67 $[0.43,1.04]$, respectively; Fig. 2; Table 2).

The pattern of changes in the annual rate of moderate-to-severe exacerbations was generally similar for the overall non-reversible and reversible subgroups, with significant benefit seen for BGF vs GFF in both populations. These findings are consistent with the changes observed overall and in non-reversible/ EOS $<300$ subgroups (Additional file 1: Table S1).

\section{Severe exacerbations}

In the overall population, BGF resulted in a nominally significant reduction in annualized severe exacerbations versus GFF, and numerical reductions versus BFF and BUD/FORM (Table 2). Similarly, BGF significantly reduced the rate of severe exacerbations versus GFF (adjusted RR [95\% CI] 0.40 [0.17, 0.94], unadjusted $p=0.0365)$ in non-reversible patients with $\mathrm{EOS}<300$ cells $/ \mathrm{mm}^{3}$, with numerical reductions versus BFF, but not versus BUD/FORM (RR [95\% CI] 0.74 [0.24, 2.30] and 2.10 [0.50, 8.81], respectively; Table 2).

In the overall non-reversible population BGF numerically reduced the rate of severe exacerbations versus GFF but not BFF or BUD/FORM (Additional file 1: Table S1). In the reversible subgroup, BGF nominally significantly reduced the rate of severe exacerbations versus GFF and BUD/FORM and numerically reduced the rate of severe exacerbations versus BFF. However, it should be noted that there were very few severe exacerbation events in the non-reversible and reversible subgroups and the results should be interpreted with caution (Additional file 1: Table S1).

\section{Safety}

Overall, safety findings in the overall population and the non-reversible with EOS $<300$ cells $/ \mathrm{mm}^{3}$ subgroup were similar (Table 3 ) and the most frequently reported treatment-emergent adverse events were nasopharyngitis, upper respiratory tract infection, COPD and bronchitis (Table 3). The incidence of confirmed pneumonia (number of patients [\%]) was similar in the overall population (32 [1.7]) and the non-reversible with EOS $<300$ cells $/ \mathrm{mm}^{3}$ subgroup (17 [1.8]).

\section{Discussion}

In this post-hoc analysis of the KRONOS study, the efficacy and safety of triple therapy with BGF 320/14.4/10 $\mu \mathrm{g}$ were evaluated in patients with moderate-to-very severe COPD who did not have reversibility to albuterol or EOS $\geq 300$ cells $/ \mathrm{mm}^{3}$, both of which are more common in patients with asthma than patients with COPD [1]. 
Table 3 Summary of TEAEs (safety population)

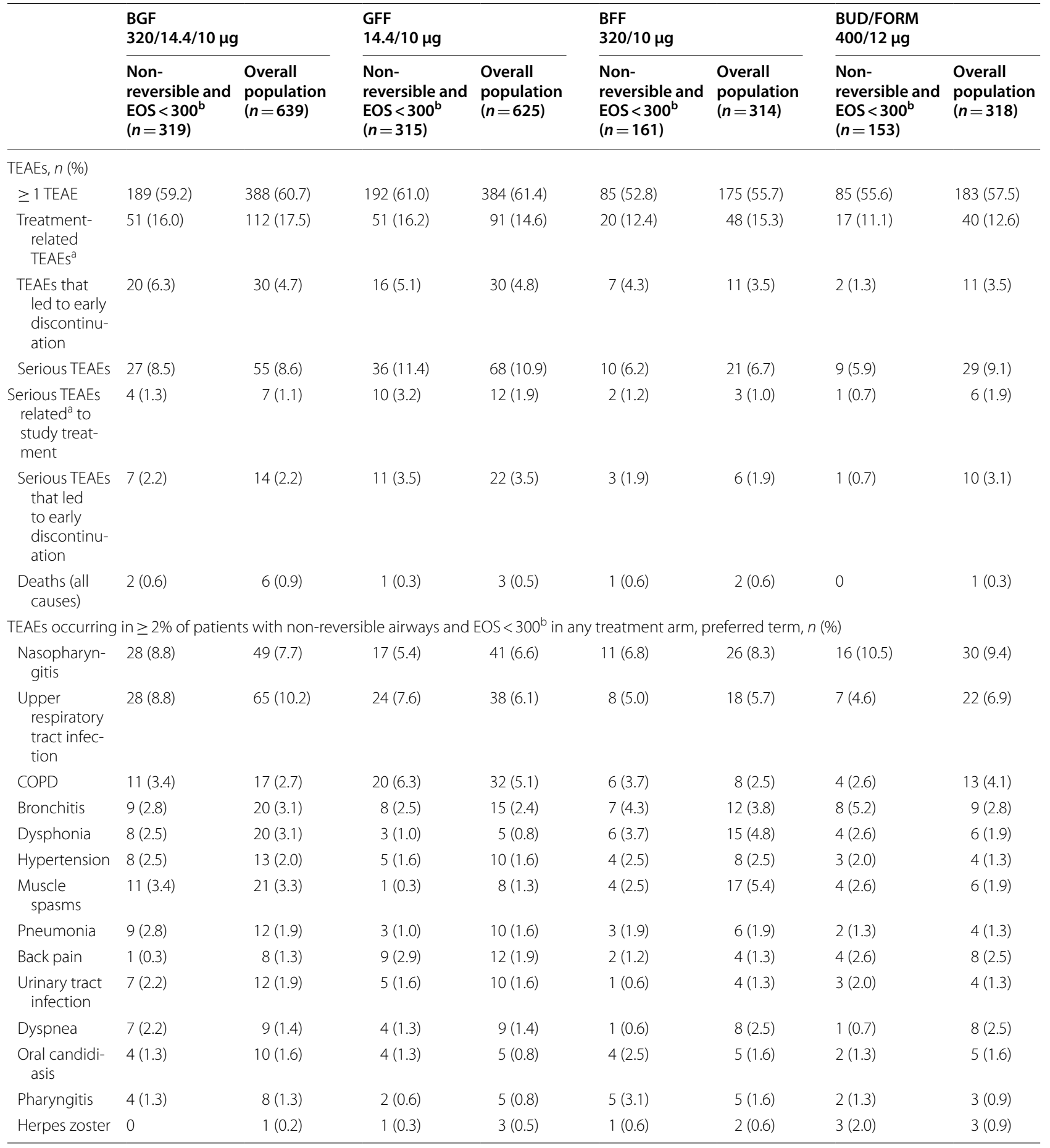

Overall population data from [4]

BFF, budesonide/formoterol fumarate dihydrate; BGF, budesonide/glycopyrronium/formoterol fumarate dihydrate; BUD/FORM DPI, budesonide/formoterol fumarate dihydrate dry powder inhaler; COPD, chronic obstructive pulmonary disease; EOS, blood eosinophil count; GFF, glycopyrronium/formoterol fumarate dihydrate; MDI, metered dose inhaler; TEAE, treatment-emergent adverse event

${ }^{\text {a }}$ Related = possibly, probably, definitely

${ }^{b}$ Patients with airways not reversible to albuterol and EOS $<300$ cells $/ \mathrm{mm}^{3}$ 
In comparison to guidelines for COPD, in which only certain patients are recommended to receive ICS-containing therapies, the Global Initiative for Asthma recommends ICS-containing therapies across all asthma severities [1, 12]. In some of the previous studies that reported benefits of triple therapy in patients with COPD, the inclusion of patients with a history of asthma has drawn criticism, with the specific concern that the observed treatment benefits may be driven by patients with asthma-like features $[5,6,13]$. However, in the KRONOS study, the benefits of ICS with BGF versus GFF were similar in patients with COPD either with or without potential asthma-like features. In both the overall population and patients whose airways were nonreversible to albuterol and who had EOS $<300$ cells $/ \mathrm{mm}^{3}$, moderate-to-severe exacerbation rates were significantly reduced for BGF compared with GFF by approximately $50 \%$ (for moderate-to-severe exacerbations) and 60\% (for severe exacerbations). A similar numerical reduction was observed in patients whose airways were reversible to albuterol; moderate-to-severe exacerbation rates were reduced for BGF compared with GFF by $55 \%$ and by approximately $80 \%$ for severe exacerbations. Eosinophils are known to impact ICS efficacy outcomes in COPD. With respect to COPD exacerbations, reductions in exacerbation rates with an ICS are observed across a broad range of eosinophil levels, with greater reductions in exacerbation rates as eosinophil levels increase [1]. With respect to lung function, improvements with an ICS are also driven by eosinophils, and, as noted previously, the improvements are seen predominantly in patients with eosinophil levels $>250$ cells $/ \mathrm{mm}^{3}$ [4]. This likely explains why lung function improvements were similar for patients receiving BGF or GFF who were non-reversible to albuterol and had EOS $<300$ cells $/ \mathrm{mm}^{3}$.

BGF resulted in significant reductions in exacerbation rates versus GFF in patients with COPD without a history of asthma and without clinical features of asthma as assessed by non-reversibility to albuterol and EOS $<300$ cells $/ \mathrm{mm}^{3}$. This is notable as current JRS guidelines recommend ICS treatment be reserved for patients with evidence of both asthma and COPD [10]. Furthermore, while the GOLD report only recommends step-up from LAMA/LABA to triple therapy in patients with continuing exacerbations [1], significant benefits of BGF vs GFF were seen despite the fact that most patients in KRONOS did not have a history of exacerbations in the previous year $(74 \%)[4,14]$. Therefore, the results of our study indicate that triple therapy may be beneficial for a wider population of patients with COPD than is reflected by current $\mathrm{ACO}$ or COPD guidelines and that the benefit of ICS on COPD exacerbations is not driven by patients with asthma-like features. The precise mechanisms underlying the reduction in exacerbations with BGF in patients without reversibility or high eosinophil levels are not known but may include enhanced bronchodilatory effects and/or anti-inflammatory effects with the triple combination relative to LAMA/LABA therapy $[1,15-$ 18]. Further, in the KRONOS study, many patients had eosinophil levels above the threshold of approximately $75-100$ cells $/ \mathrm{mm}^{3}$ at which the beneficial effects of ICS on exacerbations are manifested $[4,19]$.

Several limitations of this work should be acknowledged. These include the sample size of the subgroup of patients analyzed who were non-reversible with EOS $<300$ cells $/ \mathrm{mm}^{3}(n=948)$ and the 24-week duration of the study, which may be suboptimal when assessing COPD exacerbations and adverse events, such as pneumonia. Additionally, given their post-hoc nature, these subgroup analyses were not controlled for multiplicity. Nevertheless, we observed a clinically meaningful and significant difference between triple therapy and LAMA/LABA therapy in the subgroup of patients who were non-reversible with EOS $<300$ cells $/ \mathrm{mm}^{3}$ for both exacerbation endpoints.

\section{Conclusions}

The findings of this post-hoc analysis of patients in the KRONOS study suggest that BGF can provide benefits for a broad range of patients with COPD, including those with no evidence of concurrent common traits or clinical features of asthma. Importantly, these findings also indicate that the benefit of ICS on reducing COPD exacerbations in KRONOS was not driven by subjects with some asthma-like features.

\section{Abbreviations}

ACO: Asthma-COPD overlap; BFF: Budesonide/formoterol fumarate; BGF: Budesonide/glycopyrronium/formoterol fumarate; BUD/FORM DPI: Budesonide/formoterol fumarate dihydrate dry powder inhaler; CAT: COPD Assessment Test; Cl: Confidence interval; COPD: Chronic obstructive pulmonary disease; EOS: Blood eosinophil count; FEV 1 : Forced expiratory volume in $1 \mathrm{~s} ; \mathrm{GOLD}$ : Global Initiative for COPD; GFF: Glycopyrronium/formoterol fumarate; ICS: Inhaled corticosteroids; JRS: Japanese Respiratory Society; LABA: Long-acting $\beta_{2}$-agonists; LAMA: Long-acting muscarinic antagonists; LSM: Least squares mean; MDI: Metered dose inhaler; mITT: Modified intent-to-treat; RR: Rate ratio; SD: Standard deviation; SE: Standard error; TEAE: Treatment-emergent adverse event.

\section{Supplementary Information}

The online version contains supplementary material available at https://doi. org/10.1186/s12931-021-01773-1.

Additional file 1. Efficacy endpoints among patients with or without reversibility to albuterol, regardless of eosinophil count (mITT population, efficacy estimand). 


\section{Acknowledgements}

The authors thank all the patients and the team of investigators involved in this study. Medical writing support, under the direction of the authors, was provided by Sarah Hoyle, PhD, McCann Health Medical Communications, funded by AstraZeneca in accordance with Good Publication Practice (GPP3) guidelines [20].

\section{Authors' contributions}

The study concept and design: PDa, PDo. The acquisition, analysis, or interpretation of the data: SM, HS, PDa, PDo. The statistical analysis: PDa. The drafting of the manuscript, critical revision, and approval for submission: SM, HS, PDa, PDo.

\section{Funding}

This study was supported by AstraZeneca.

\section{Availability of data and materials}

Data underlying the findings described in this manuscript may be obtained in accordance with AstraZeneca's data sharing policy described at https://astra zenecagrouptrials.pharmacm.com/ST/Submission/Disclosure

\section{Declarations}

\section{Ethics approval and consent to participate}

This study was done in accordance with Good Clinical Practice, including the Declaration of Helsinki. The protocol and informed consent form were approved by appropriate institutional review boards or independent ethics committees [4]. All patients provided written informed consent before screening.

\section{Consent for publication}

Not applicable.

\section{Competing interests}

SM has received lecture fees from AstraZeneca, GSK, Nippon Boehringer Ingelheim, and Novartis Pharma. HS has received lecture fees from AstraZeneca, GSK, Nippon Boehringer Ingelheim, and Novartis Pharma. PDo is an employee of AstraZeneca and holds stock and/or stock options in the company. PDa is an employee of AstraZeneca and holds stock and/or stock options in the company.

\section{Author details}

'Department of Respiratory Medicine, Nara Medical University Graduate School of Medicine, 840 Shijo-cho, Kashihara-shi, Nara 634-8521, Japan. ${ }^{2}$ Department of Respiratory Medicine, Tohoku University Graduate School of Medicine, Sendai, Japan. ${ }^{3}$ AstraZeneca, Wilmington, DE, USA. ${ }^{4}$ AstraZeneca, Durham, NC, USA.

Received: 7 April 2021 Accepted: 6 June 2021

Published online: 28 June 2021

\section{References}

1. Global Initiative for Chronic Obstructive Lung Disease. Global strategy for the diagnosis, management, and prevention of chronic obstructive pulmonary disease report. 2020. https://goldcopd.org/wp-content/uploa ds/2019/11/GOLD-2020-REPORT-ver1.0wms.pdf. Accessed 2 Mar 2021.

2. Yanagisawa $S$, Ichinose M. Definition and diagnosis of asthma-COPD overlap (ACO). Allergol Int. 2018;67(2):172-8.

3. Sin DD, Miravitlles M, Mannino DM, Soriano JB, Price D, Celli BR, et al. What is asthma-COPD overlap syndrome? Towards a consensus definition from a round table discussion. Eur Respir J. 2016;48(3):664-73.

4. Ferguson GT, Rabe KF, Martinez FJ, Fabbri LM, Wang C, Ichinose M, et al. Triple therapy with budesonide/glycopyrrolate/formoterol fumarate with co-suspension delivery technology versus dual therapies in chronic obstructive pulmonary disease (KRONOS): a double-blind, parallel-group, multicentre, phase 3 randomised controlled trial. Lancet Respir Med. 2018;6(10):747-58.

5. Suissa S, Ariel A. Triple therapy trials in COPD: a precision medicine opportunity. Eur Respir J. 2018;52(6):1801848.

6. Vanfleteren L, Fabbri LM, Papi A, Petruzzelli S, Celli B. Triple therapy (ICS/ LABA/LAMA) in COPD: time for a reappraisal. Int J Chron Obstruct Pulmon Dis. 2018;13:3971-81.

7. Rabe KF, Martinez FJ, Ferguson GT, Wang C, Singh D, Wedzicha JA, et al. Exacerbation benefit by blood eosinophil counts with budesonide/glycopyrronium/formoterol metered dose inhaler (BGF MDI) at two ICS dose levels in the ETHOS trial: a subgroup analysis. Eur Respir J. 2020;56(suppl 64):984.

8. Fabbri LM, Rabe KF, Martinez FJ, Ferguson GT, Wang C, Ichinose M, et al. Late breaking abstract-impact of eosinophil levels on lung function and exacerbation benefits with co-suspension delivery technology budesonide/glycopyrronium/formoterol metered dose inhaler (BGF MDI) in COPD (KRONOS study subgroup analysis). Eur Respir J. 2018;52(62):PA688.

9. Donohue JF, Ohar JA. Effects of corticosteroids on lung function in asthma and chronic obstructive pulmonary disease. Proc Am Thorac Soc. 2004;1(3):152-60.

10. The Japanese Respiratory Society. Guidelines for the Diagnosis and Treatment of COPD, 5th Edition (in Japanese). 2018. http://www.jrs.or.jp/ modules/guidelines/index.php?content_id=112. Accessed 26 Feb 2019.

11. Sasaki H, Nakamura M, Kida K, Kambe M, Takahashi K, Fujimura M, et al. Reference values for spirogram and blood gas analysis in Japanese adults. J Jpn Respir Soc. 2001;39(5):S1-17.

12. Global Initiative for Asthma. Global strategy for asthma management and prevention. 2020. https://ginasthma.org/gina-reports/. Accessed 4 May 2020.

13. Suissa S, Drazen JM. Making sense of triple inhaled therapy for COPD. N Engl J Med. 2018;378(18):1723-4.

14. Martinez FJ, Ferguson GT, Bourne E, Ballal S, Darken P, Aurivillius M, et al. Budesonide/glycopyrrolate/formoterol fumarate metered dose inhaler improves exacerbation outcomes in patients with COPD without a recent exacerbation history: a subgroup analysis of KRONOS. Int J Chron Obstruct Pulmon Dis. 2021;16:179-89.

15. Barrette AM, Roberts JK, Chapin C, Egan EA, Segal MR, Oses-Prieto JA, et al. Antiinflammatory effects of budesonide in human fetal lung. Am J Respir Cell Mol Biol. 2016;55(5):623-32.

16. Hakim A, Khan Y, Esteban I, Meah S, Miller-Larsson A, Barnes PJ, et al. Low-dose budesonide/formoterol counteracts airway inflammation and improves lung function in chronic obstructive pulmonary disease. Am J Respir Crit Care Med. 2019;199(5):662-4.

17. Mokra D, Kosutova P, Balentova S, Adamkov M, Mikolka P, Mokry J, et al. Effects of budesonide on the lung functions, inflammation and apoptosis in a saline-lavage model of acute lung injury. J Physiol Pharmacol. 2016;67(6):919-32

18. Usmani OS, Ito K, Maneechotesuwan K, Ito M, Johnson M, Barnes PJ, et al. Glucocorticoid receptor nuclear translocation in airway cells after inhaled combination therapy. Am J Respir Crit Care Med. 2005;172(6):704-12.

19. Bafadhel M, Peterson S, De Blas MA, Calverley PM, Rennard SI, Richter $K$, et al. Predictors of exacerbation risk and response to budesonide in patients with chronic obstructive pulmonary disease: a post-hoc analysis of three randomised trials. Lancet Respir Med. 2018;6(2):117-26.

20. Battisti WP, Wager E, Baltzer L, Bridges D, Cairns A, Carswell Cl, et al. Good publication practice for communicating company-sponsored medical research: GPP3. Ann Intern Med. 2015;163(6):461-4.

\section{Publisher's Note}

Springer Nature remains neutral with regard to jurisdictional claims in published maps and institutional affiliations. 